7 Running title: Force depression in Type I and Type II fibers

8

11 Word count (Introduction-Acknowledgements) $=3492$

12 Corresponding Author:

13 Dr. Venus Joumaa

14 University of Calgary, 2500 University Dr. N.W., Calgary, AB, Canada, T2N 1N4

15

16

17

\section{skeletal muscle}

\author{
V. Joumaa, G.A. Power, B. Hisey, A. Caicedo, J. Stutz and W. Herzog \\ Human Performance Laboratory, University of Calgary, Alberta, Canada
}

Keywords: MHC, Mechanical work, Speed of shortening, Transient forces, Stiffness, Historydependence, Cross-bridge theory, Fast-twitch, Slow-twitch

\title{
Effects of fiber type on force depression after active shortening in
}




\section{$18 \underline{\text { Abstract }}$}

19 The aim of this study was to investigate force depression in Type I and Type II muscle fibers.

20 Experiments were performed using skinned fibers from rabbit soleus and psoas muscles. Force

21 depression was quantified after active fiber shortening from an average sarcomere length (SL) of

$223.2 \mu \mathrm{m}$ to an average SL of $2.6 \mu \mathrm{m}$ at an absolute speed of 0.115 fiber length/s and at a relative

23 speed corresponding to $17 \%$ of the unloaded shortening velocity $\left(\mathrm{V}_{0}\right)$ in each type of fibers.

24 Force decay and mechanical work during shortening were also compared between fiber types.

25 After mechanical testing, each fiber was subjected to myosin heavy chain (MHC) analysis in

26 order to confirm its Type (Type I expressing MHC I, and Type II expressing MHC IId). Type II

27 fibers showed greater steady-state force depression after active shortening at a speed of 0.115

28 fiber length/s than Type I fibers $(14.5 \pm 1.5 \%$ versus $7.8 \pm 1.7 \%)$. Moreover, at this absolute

29 shortening speed, Type I fibers showed a significantly greater rate of force decay during

30 shortening and produced less mechanical work than Type II fibers. When active shortening was

31 performed at the same relative speed $\left(17 \% \mathrm{~V}_{0}\right)$, the difference in force depression between fiber

32 types was abolished. These results suggest that no intrinsic differences were at the origin of the

33 difference in force depression observed in Type I and Type II fibers when actively shortened at

34 the same speed, but rather their distinct force-velocity relationships. 


\section{Introduction}

36 The steady-state isometric force after active shortening of skeletal muscle is smaller than the

37 purely isometric force at the same level of activation and the corresponding length. This

38 phenomenon, referred to as force depression, was first described by Abbott and Aubert in 1952

39 and has been observed consistently in whole and isolated muscle preparations (Rassier and

40 Herzog, 2004). The molecular mechanisms causing force depression remain unknown, but two

41 primary mechanisms have been suggested. First, force depression has been associated with the

42 development of sarcomere length non-uniformities upon active shortening (Morgan et al., 2000).

43 According to this theory, force depression is caused by the development of large dispersions in

44 sarcomere lengths during active shortening. Second, force depression has been associated with a

45 stress-induced inhibition of cross bridges in the newly formed actin-myosin overlap zone

46 following shortening owing to actin angular deformation (Marechal and Plaghki, 1979).

47 According to this theory, active shortening causes a decrease in the probability of cross bridge

48 attachment, thereby leading to a decrease in the proportion of force generating (strongly bound)

49 cross bridges in the force depressed state at the same muscle length and overlap of thick and thin

50 filaments compared to a purely isometric contraction.

51 Despite an abundance of information regarding the characteristics and mechanisms of force

52 depression, there has been no investigation comparing force depression among skeletal muscle

53 fiber types. Fast (Type II) and slow (Type I) muscle fibers have distinctly different force-velocity

54 properties (Bottinelli et al., 1991), and thus force and work for shortening at a given speed differs

55 substantially between fiber types. It has been shown that the magnitude of force depression is 56 directly related to force and work performed during shortening (Corr and Herzog, 2005;

57 Dargeviciute et al., 2013; Leonard and Herzog, 2005; Minozzo and Rassier, 2013). 
58 The aim of the present study was to investigate force depression in Type I and Type II muscle

59 fibers. Based on the acknowledged differences in force-velocity properties and transient forces

60 between fiber types (Bottinelli et al., 1991), we hypothesized that force depression following

61 active shortening at a given absolute speed is greater in Type II than Type I fibers but would be 62 similar between fiber types for similar relative speed of shortening. 


\section{Materials and methods}

64 New Zealand white rabbits were euthanized according to a protocol approved by the University

65 of Calgary's Animal Care and Ethics Committee. Strips of soleus and psoas muscles were

66 skinned and fibers were isolated and mounted between a force transducer and a length controller

67 as previously described (Joumaa and Herzog, 2013; Toursel et al., 2000). SLs were measured

68 using optical diffraction of a He-Ne laser beam. Fiber cross sectional area and volume were

69 calculated assuming the fiber has a cylindrical shape. Experiments were performed at $15^{\circ} \mathrm{C}$.

Maximal stress at an average SL of $2.6 \mu \mathrm{m}$

71 Soleus $(n=15)$ and psoas $(n=22)$ fibers were activated at an average SL of $2.6 \mu \mathrm{m}$ in order to

72 measure their maximal force at this length. Force was normalized to the fiber cross sectional area

73 to obtain the maximal stress.

74 Active shortening contractions at a shortening speed of 0.115 fiber length/s

75 Soleus $(n=15)$ and psoas $(n=15)$ fibers were set at an average SL of $2.6 \mu \mathrm{m}$ and then passively

76 stretched to an average SL of $3.2 \mu \mathrm{m}$, held for 20 seconds and activated by changing the relaxing

77 solution to a high calcium activating solution (Joumaa and Herzog, 2013). Fibers were then

78 actively shortened to an average SL of $2.6 \mu \mathrm{m}$ at a speed of 0.115 fiber length/s, held 79 isometrically until steady-state isometric force was reached, and then deactivated (Figure 1). 80 After a rest period of 5 minutes, fibers were activated at an average SL of $2.6 \mu \mathrm{m}$ in order to 81 measure their purely isometric reference force.

82 Active shortening contractions at the same relative speed between Type I and Type II fibers 
83 In order to perform active shortening at the same relative speed between Type I and Type II

84 fibers, we measured the unloaded shortening velocity $\left(V_{0}\right)$ for psoas $(n=5)$ and soleus $(n=5)$

85 fibers using the slack test method proposed by Edman (1979). Then we tested an additional 86 group of psoas fibers in which we performed active shortening at the same relative speed $\left(\% \mathrm{~V}_{0}\right)$

87 as in soleus fibers. The unloaded shortening velocities were (mean \pm SEM) $0.67 \pm 0.09$ fiber 88 length/s and 1.77 \pm 0.03 fiber length/s in soleus and psoas fibers, respectively. The initial active 89 shortening experiments were performed at a shortening speed of 0.115 fiber length/s 90 corresponding to a relative shortening speed of $17 \%$ of $\mathrm{V}_{0}$ in Type $\mathrm{I}$ fibers. Thus, seven 91 additional psoas fibers were actively shortened at a relative speed of $17 \%$ of their $\mathrm{V}_{0}$ 92 corresponding to 0.3 fiber length/s.

\section{Stiffness measurements}

94 Fiber stiffness was obtained using a quick stretch-release protocol of $0.2 \%$ fiber length (Ford et 95 al., 1981; Joumaa et al., 2012; Mansson, 1989; Rassier and Herzog, 2005) at a speed of 1 fiber 96 length/s. Stiffness was measured once the isometric steady-state had been reached after active 97 shortening, and during the purely isometric reference contraction.

MHC content

99 The MHC content was determined in each fiber after mechanical testing using SDS-PAGE gel 100 electrophoresis on $4.5 \%$ and $7.5 \%$ acrylamide stacking and separating gels respectively (Toursel 101 et al., 2000). The gels were run in a Biorad Mini-Protean III unit at a constant voltage of $73 \mathrm{~V}$ for 10240 hours at $4^{\circ} \mathrm{C}$, stained with Coomassie Blue and scanned. Following electrophoresis, fibers 103 were divided into two groups: Type I fibers expressing MHC I and Type II fibers expressing 104 MHC IId. 
105

106

107

108

109

110

111 amplitude of the stretch.

112 Rate of force decline during shortening: Force during shortening is characterized by a steep

113 initial phase and a slow final phase. The initial and final phases were fitted by two linear least-

114 squares regression functions. The slope of the initial steep phase of shortening, S1, was taken as

115 an estimate of the rate of force decline during shortening. The slope of the final slow phase of

116 shortening, S2, was taken as an estimate of the behaviour of the non-contractile (passive)

117 element during shortening (Roots et al., 2007). S1 and S2 were normalized to the maximal

118 isometric fiber force and compared between Type I and Type II fibers.

119 Mechanical work: Mechanical work during shortening was calculated by trapezoidal numerical 120 integration of the force-length curve during the shortening phase. To compare work performed 121 between fibers, the mechanical work was normalized to fiber volume.

122 Statistical analysis: All data reported are means \pm SEM. The Student's $t$ test $(\mathrm{p}<0.05)$ was used to 123 compare data between Type I and Type II fibers. 


\section{$124 \quad$ Results}

125 Fibers were analysed for MHC composition (Figure 2). Thirteen fibers isolated from the soleus 126 muscle and tested for force depression expressed MHC I and were grouped as Type I fibers. The 127 remaining two soleus fibers expressed MHC IIa and were excluded from the analysis. Three 128 psoas fibers were excluded from analysis because they co-expressed MHCs IId/IIb. The 129 remaining psoas fibers tested for force depression $(n=12$ shortened at a speed of 0.115 fiber 130 length/s, $\mathrm{n}=7$ shortened at a relative speed of $17 \% \mathrm{~V}_{0}$ ) expressed MHC IId and were labelled 131 Type II fibers and analyzed.

132 The maximal stress at an average SL of $2.6 \mu \mathrm{m}$ for Type I fibers $\left(151 \pm 14 \mathrm{kN} / \mathrm{m}^{2}\right)$ was not 133 different from that of Type II fibers $\left(159 \pm 13 \mathrm{kN} / \mathrm{m}^{2}\right)$.

134 Active shortening contractions at a shortening speed of 0.115 fiber length/s

135 Force depression in Type II fibers was higher than that of Type I fibers $(14.5 \pm 1.5 \%$ versus $1367.8 \pm 1.7 \%$ ) when fibers were shortened at the same absolute speed of 0.115 fiber length/s. Type I 137 fibers showed a steeper decay in force during shortening (Table 1, Figure 3), performed less 138 mechanical work during shortening and showed smaller S2 than Type II fibers (Table 1).

139 Active shortening contractions at the same relative speed between Type I and Type II fibers

140 When Type II fibers were shortened at the same relative speed as Type I fibers $\left(17 \% \mathrm{~V}_{0}\right)$, the 141 difference in force depression between fiber types was abolished $(4.1 \pm 1.8 \%$ in Type II versus $1427.8 \pm 1.7 \%$ in Type I). Furthermore, the mechanical work and S1 were not different between fiber 143 types (Table 1), but S2 was still smaller in Type I fibers compared to Type II

144 Force depression was correlated with stiffness depression $\left(R^{2}=0.6, p<0.05\right.$, Figure 4) and $S 1$ $145 \quad\left(\mathrm{R}^{2}=0.4, \mathrm{p}<0.05\right.$, Figure 5$)$ across all fibers. 


\section{Discussion}

147 Our main finding in this study is that Type II fibers when actively shortened at the same absolute

148 speed showed greater isometric steady-state force depression after active shortening compared to

149 Type I fibers. However, the difference in force depression between fiber types was abolished 150 when active shortening was performed at the same relative speed suggesting that no intrinsic 151 differences were at the origin of the different force depression in Type I and Type II fibers, but 152 rather their different force-velocity relationships.

153 The force-velocity relationship is markedly different between muscle fiber types (Bottinelli et al., 154 1991). Type I fibers have lower maximal shortening velocities $\left(\mathrm{V}_{\max }\right)$ and a greater curvature of 155 their force-velocity relationship compared to Type II fibers. When active shortening in the 156 present study was performed at the same absolute speed (relative to fiber length) in Type I and 157 Type II fibers, Type I fibers were shortening at a higher speed relative to their $\mathrm{V}_{\max }$, and thus 158 produced less force during shortening than Type II fibers. A decreased force over the given 159 displacement results in a lower mechanical work performed during shortening in Type I fibers as 160 compared with Type II fibers. The increased rate of force decay in conjunction with the 161 decreased mechanical work performed during shortening observed for Type I fibers provide 162 quantitative support for the rationale that force and work during shortening are reduced in Type I 163 compared with Type II fibers owing to their intrinsic force-velocity properties.

164 It has been found that force during the active shortening phase is highly correlated with the 165 steady-state isometric force reached after shortening (Dargeviciute et al., 2013; Leonard and 166 Herzog, 2005). Leonard and Herzog (2005) showed that force depression was the same when 167 force during shortening was kept constant, even though the speed of shortening was changed 168 substantially. Conversely, they found that force depression varied greatly when force during 
169 shortening was altered while shortening speed was kept constant (Herzog and Leonard, 1997).

170 Furthermore, since force depression is associated with the magnitude of shortening and force

171 during shortening, it has been demonstrated that force depression is directly proportional to the

172 mechanical work performed during shortening (Dargeviciute et al., 2013; Herzog and Leonard,

173 1997; Leonard and Herzog, 2005). Therefore, the lower force along with the decreased

174 mechanical work performed by Type I compared with Type II fibers explains the decreased force 175 depression in Type I compared to Type II fibers.

176 It has been hypothesized that since thin and thick filaments are slightly compliant (Goldman and 177 Huxley, 1994; Kojima et al., 1994), when muscle is activated, mechanical stress causes a small 178 elongation of the thin filament, possibly resulting in an angular distortion of the myosin binding 179 sites on actin. This distortion may cause a decrease in the attachment rate of cross bridges and 180 thus an inhibition of their formation in the newly formed overlap zone when muscle is then 181 shortened and the stress in thin filaments is maintained. A decreased speed of shortening is 182 associated with increased force, thus a potential increase in the deformation of actin and 183 increased force depression. According to Marechal and Plaghki (1979), force depression "is 184 precisely controlled by the mechanical parameters of the release", when the load - tetanic stress 185 is light, cross bridges are slightly inhibited and when the load is heavy, cross bridges are partially 186 or completely inhibited. The relationship between force depression and stiffness depression 187 found here (Figure 4) and by others (Minozzo and Rassier, 2013; Rassier and Herzog, 2004) 188 supports the stress induced inhibition of cross bridge theory.

189 The sarcomere length non-uniformity theory could potentially explain the difference in force 190 depression between fiber types when actively shortened at the same absolute speed. Because 191 sarcomeres are assumed to be unstable on the descending limb of the force length relationship 
192 (Hill, 1953) regardless of the fiber type, we suggest that sarcomere length non-uniformities 193 develop in Type I and Type II fibers. To our knowledge, the amount of sarcomere length non194 uniformities has not been compared between fast and slow fibers. However, if we assume that 195 the development of sarcomere length non-uniformities increases during length changes when 196 force is high, we might expect greater non-uniformities in sarcomere lengths in fast compared to 197 slow fibers and thus a greater amount of force depression when shortening at the same absolute 198 speed. However, experiments in single myofibrils and sarcomeres have shown that the 199 development of sarcomere length non-uniformities is likely not the mechanism for force 200 depression (Joumaa and Herzog, 2010; Trecarten et al., 2015).

201 Passive properties are different between Type I and type II fibers. Rabbit psoas muscle has 202 shorter titin isoforms (two isoforms of molecular weights of $~ 3.3$ and $3.4 \mathrm{MDa}$ ) compared to 203 titin isoform in the rabbit soleus muscle (one titin isoform of a molecular weight of 3.6 MDa) 204 (Prado et al., 2005). Could this difference in titin isoforms be responsible for the difference in 205 force depression observed at an average sarcomere length of $2.6 \mu \mathrm{m}$ between Type I and Type II 206 fibers? According to the primary mechanisms of force depression (i.e. the stress induced 207 inhibition of cross bridges and the non-uniformity theory), passive force is not involved in force 208 depression. However, Leonard and Herzog (2010) proposed a mechanism for residual force 209 enhancement based on the interaction between (binding of) titin and actin upon 210 muscle/fiber/myofibril activation. When the myofibril is stretched, its passive force is increased 211 because the strain on titin is higher compared to a purely isometric contraction. Could titin's role 212 in force depression mirror that of force enhancement? Indeed, Rode et al. (2009) suggested, 213 based on a theoretical model using Hill-type muscle and incorporating titin as a "sticky spring", 214 that titin might play a major role in force depression. Rode et al. (Rode et al., 2009) proposed 
215 that titin attaches to the actin filament upon muscle activation. As a result, the length of titin's 216 free spring length is dramatically reduced. This leads to an increased passive force when the 217 sarcomere is stretched and a decreased passive force when the sarcomere is shortened. Based on 218 this model, one might suggest that the shorter the titin isoform, the higher the reduction in force 219 at steady-state following sarcomere shortening. Although many aspects of the "sticky-spring" 220 mechanism should be carefully considered (i.e. the (non-) selective binding of titin to actin in 221 long and short isoforms, the region of titin that binds to actin, the behaviour of titin during 222 shortening, etc.), this mechanism could potentially explain the greater force depression observed 223 in psoas Type II fibers compared to soleus Type I fibers. Titin contributions to force depression 224 have also been proposed by Nishikawa et al. (2012) who suggested that titin might be 225 responsible for force depression by a mechanism of winding and unwinding of titin onto the 226 rotating actin filament. According to Roots et al. (2007), the contribution of the non-contractile 227 (passive) element to force during shortening can be determined by S2 phase during shortening. 228 S2 in Type I fibers was lower than that in Type II fibers suggesting that the passive element 229 behaviour is different during shortening between fiber types. Passive force decreased at a higher 230 rate during shortening in the Type II compared to Type I fibers. This finding agrees with the 231 "sticky-spring" mechanism (Rode et al., 2009) for force depression in that short titin isoforms 232 would be predicted to show increased rates of force reduction during shortening compared to 233 long titin isoforms. Therefore, the diversity in titin isoforms between fiber types could 234 potentially account for their differences in force depression.

235 In addition to their diversity in myosin heavy chain and titin isoforms, skeletal muscle fiber types 236 also show numerous differences in protein structures (actin, troponin, tropomyosin, etc.) and 237 functional properties (ATPase hydrolysis rate, cross bridge cycling rate, etc.). Therefore, it is not 
238 clear whether force depression is independently related to structural differences between fiber 239 types, or if fiber types show different force depressions exclusively because of the difference in 240 their force-velocity relationships. In our tests at a fixed absolute speed of shortening, we found 241 that force depression was greater in Type II compared to Type I fibers. Shortening at the same 242 absolute speed resulted in Type I fibers shortening at a higher speed relative to their $\mathrm{V}_{\max }$ and 243 correspondingly a lower force than Type II fibers. In order to test if the difference in force 244 depression between Type I and Type II fibers was an intrinsic property of fiber types, we 245 determined the unloaded shortening velocity for each fiber type and force depression was then 246 determined for conditions of the same relative speed of shortening for both fiber types. When the 247 shortening speed relative to $\mathrm{V}_{0}$ was the same for the two fiber types, the differences in force 248 depression were abolished, suggesting that no intrinsic fiber type differences were the cause for 249 the differences in force depressions observed when shortening at the same absolute speed. In the 250 experiments performed at the same relative speed, S2 was lower in Type I fibers compared to 251 Type II fibers, suggesting that the behaviour of the passive element during shortening is not 252 responsible for force depression at the steady-state after shortening.

253 According to the stress-induced inhibition of cross bridge theory (Marechal and Plaghki, 1979), 254 force production prior to active shortening is assumed to cause extension and distortion of the 255 thin filaments. Thin filament distortion is thought to be proportional to its compliance; the higher 256 the compliance, the higher the distortion (Herzog and Leonard, 1997; Kojima et al., 1994). When 257 shortening occurred with similar forces between Type I and Type II fibers, force depression was 258 also similar between fiber types. Thus it appears that the compliance of thin filaments of Type II 259 fibers is similar to that of Type I fibers. This speculation is in accordance with previous findings 260 (Galler and Hilber, 1998) showing that the variations in the structure of proteins between fiber 
261 types are not sufficient to produce different compliances of the network of proteins within 262 sarcomeres.

263 Why do Type I fibers exhibit less force than Type II fibers during shortening, while the opposite 264 happens at the steady-state after shortening? Different mechanisms control transient muscle force 265 during shortening and isometric force after shortening. Force decreases during active isokinetic 266 shortening because of a decrease in the number of attached cross bridges and in the amount of 267 force produced per cross bridge (Huxley, 1957). It is known that fast fibers have high rates of 268 ATP hydrolysis and cross bridge attachment and therefore as filaments slide past one another at 269 the same speed for fast and slow fibers, fewer cross bridges have the time to attach in slow fibers 270 and thus force decreases more in slow compared to fast fibers (Barany et al., 1965). Force per 271 cross bridge decreases during shortening because the distance " $x$ " (Huxley, 1957) decreases 272 when the filaments slide past one another. Since it has been suggested that the stiffness of the 273 elastic region of the cross bridge might be higher in Type I compared to Type II fibers (Goubel 274 and Marini, 1987; Pousson et al., 1991), the decrease in force for the same decrease in " $x$ " 275 distance would be higher in Type I compared to Type II fibers. Accordingly, the relatively lesser 276 decrease in force during isokinetic shortening of Type II fibers compared to Type I fibers is 277 likely due to a smaller decrease in the number of attached cross bridges and a smaller decrease in 278 the force produced per cross bridge. On the other hand, in the isometric force depressed state, the 279 primary mechanisms are, as discussed earlier, the stress induced inhibition of cross bridges 280 (Marechal and Plaghki, 1979) and the non-uniformity theory (Morgan et al., 2000).

281 The biomechanical role of force depression during everyday movement is still unknown (Tilp et 282 al., 2009). However, it has been speculated that active shortening and force reduction during 283 shortening could reduce the work required to induce active lengthening (negative work) and thus, 
284 increase the net work performed by a muscle over a full cyclic shortening/lengthening movement 285 (Josephson and Stokes, 1999).

286 In line with the stress-induced theory of force depression, we show for the first time greater force 287 depression in Type II fast muscle fibers compared with Type I slow muscle fibers when 288 shortening was performed at the same absolute speed. When shortening speed was controlled and 289 Type II and Type I shortened at the same relative speed, no difference in force depression was 290 observed.

291 
292 Acknowledgements

293 CIHR, NSERC, Canada Research Chair Programme. G.A. Power is supported by a Banting 294 postdoctoral fellowship (CIHR) and Alberta Innovates Health Solutions (AIHS). 


\section{References}

Barany, M., Barany, K., Reckard, T., Volpe, A., 1965. Myosin of Fast and Slow Muscles of the Rabbit. Arch Biochem Biophys 109, 185-91.

Bottinelli, R., Schiaffino, S., Reggiani, C., 1991. Force-velocity relations and myosin heavy chain isoform compositions of skinned fibres from rat skeletal muscle. J Physiol 437, $655-72$.

Corr, D.T., Herzog, W., 2005. Force recovery after activated shortening in whole skeletal muscle: transient and steady-state aspects of force depression. J Appl Physiol (1985) 99, $252-60$.

Dargeviciute, G., Masiulis, N., Kamandulis, S., Skurvydas, A., Westerblad, H., 2013. Residual force depression following muscle shortening is exaggerated by prior eccentric drop jump exercise. J Appl Physiol (1985) 115, 1191-5.

Edman, K.A., 1979. The velocity of unloaded shortening and its relation to sarcomere length and isometric force in vertebrate muscle fibres. J Physiol 291, 143-59.

Ford, L.E., Huxley, A.F., Simmons, R.M., 1981. The relation between stiffness and filament overlap in stimulated frog muscle fibres. J Physiol 311, 219-49.

Galler, S., Hilber, K., 1998. Tension/stiffness ratio of skinned rat skeletal muscle fibre types at various temperatures. Acta Physiol Scand 162, 119-26.

Goldman, Y.E., Huxley, A.F., 1994. Actin compliance: are you pulling my chain? Biophys J 67, 2131-3.

Goubel, F., Marini, J.F., 1987. Fibre type transition and stiffness modification of soleus muscle of trained rats. Pflugers Arch 410, 321-5. 
317 Herzog, W., Leonard, T.R., 1997. Depression of cat soleus-forces following isokinetic shortening. J Biomech 30, 865-72.

319 Hill, A.V., 1953. The mechanics of active muscle. Proc R Soc Lond B Biol Sci 141, 104-17.

320 Horowits, R., 1992. Passive force generation and titin isoforms in mammalian skeletal muscle. Biophys J 61, 392-8.

322 Huxley, A.F., 1957. Muscle structure and theories of contraction. Prog Biophys Biophys Chem 323 7, 255-318.

324 Josephson, R.K., Stokes, D.R., 1999. Work-dependent deactivation of a crustacean muscle. J Exp 325 Biol 202, 2551-2565.

326 Joumaa, V., Herzog, W., 2010. Force depression in single myofibrils. J Appl Physiol 108, 356327 62.

328 329 330 331 332 333 334 335 336 337 338
Joumaa, V., Herzog, W., 2013. Energy cost of force production is reduced after active stretch in skinned muscle fibres. J Biomech 46, 1135-9.

Joumaa, V., Macintosh, B.R., Herzog, W., 2012. New insights into force depression in skeletal muscle. J Exp Biol 215, 2135-40.

Kojima, H., Ishijima, A., Yanagida, T., 1994. Direct measurement of stiffness of single actin filaments with and without tropomyosin by in vitro nanomanipulation. Proc Natl Acad Sci U S A 91, 12962-6.

Leonard, T.R., Herzog, W., 2005. Does the speed of shortening affect steady-state force depression in cat soleus muscle? J Biomech 38, 2190-7.

Leonard, T.R., Herzog, W., 2010. Regulation of muscle force in the absence of actin-myosinbased cross-bridge interaction. Am J Physiol Cell Physiol 299, C14-20. 
339 Mansson, A., 1989. Changes in force and stiffness during stretch of skeletal muscle fibers, 340 effects of hypertonicity. Biophys J 56, 429-33.

341

342

343

344

345

346

347

348

349

350

351

352

353

354

355

356

357

358

359

360

Marechal, G., Plaghki, L., 1979. The deficit of the isometric tetanic tension redeveloped after a release of frog muscle at a constant velocity. J Gen Physiol 73, 453-67.

Minozzo, F.C., Rassier, D.E., 2013. The effects of Ca2+ and MgADP on force development during and after muscle length changes. PLoS One 8, e68866.

Morgan, D.L., Whitehead, N.P., Wise, A.K., Gregory, J.E., Proske, U., 2000. Tension changes in the cat soleus muscle following slow stretch or shortening of the contracting muscle. $\mathrm{J}$ Physiol 522 Pt 3, 503-13.

Nishikawa, K.C., Monroy, J.A., Uyeno, T.E., Yeo, S.H., Pai, D.K., Lindstedt, S.L., 2012. Is titin a 'winding filament'? A new twist on muscle contraction. Proc Biol Sci 279, 981-90.

Pousson, M., Perot, C., Goubel, F., 1991. Stiffness changes and fibre type transitions in rat soleus muscle produced by jumping training. Pflugers Arch 419, 127-30.

Prado, L.G., Makarenko, I., Andresen, C., Kruger, M., Opitz, C.A., Linke, W.A., 2005. Isoform diversity of giant proteins in relation to passive and active contractile properties of rabbit skeletal muscles. J Gen Physiol 126, 461-80.

Rassier, D.E., Herzog, W., 2004. Considerations on the history dependence of muscle contraction. J Appl Physiol 96, 419-27.

Rassier, D.E., Herzog, W., 2005. Relationship between force and stiffness in muscle fibers after stretch. J Appl Physiol 99, 1769-75.

Rode, C., Siebert, T., Blickhan, R., 2009. Titin-induced force enhancement and force depression: a 'sticky-spring' mechanism in muscle contractions? J Theor Biol 259, 350-60. 
361 Roots, H., Offer, G.W., Ranatunga, K.W., 2007. Comparison of the tension responses to ramp 362 shortening and lengthening in intact mammalian muscle fibres: crossbridge and noncrossbridge contributions. J Muscle Res Cell Motil 28, 123-39.

364 Tilp, M., Steib, S., Herzog, W., 2009. Force-time history effects in voluntary contractions of 365 human tibialis anterior. Eur J Appl Physiol 106, 159-66.

366 Toursel, T., Bastide, B., Stevens, L., Rieger, F., Mounier, Y., 2000. Alterations in contractile 367 properties and expression of myofibrillar proteins in wobbler mouse muscles. Exp Neurol $162,311-20$.

369 Trecarten, N., Minozzo, F.C., Leite, F.S., Rassier, D.E., 2015. Shortening-induced force 370 depression in single sarcomeres is abolished by MgADP-activation. Biophys J 108, 338a. 


\section{$372 \underline{\text { Table } 1}$}

373

374

375

376

377

378

379 Mean values \pm SEM for S1, S2 and the mechanical work performed during shortening at the

380 absolute and relative speeds. * indicates a significant difference of Type I fibers compared to

381 Type II fibers shortened at the same absolute speed of 0.115 fiber length $/ \mathrm{s}(\mathrm{p}<0.05),{ }^{\|}$indicates a

382 significant difference of Type I fibers compared to Type II fibers shortened at the same relative 383 speed of $17 \% \mathrm{~V}_{0}(\mathrm{p}<0.05)$. 


\section{$385 \quad$ Figure captions}

386 Figure 1

387 Typical fiber response when passively stretched from an average SL of $2.6 \mu \mathrm{m}$ to an average SL 388 of $3.2 \mu \mathrm{m}$, activated, shortened to an average SL of $2.6 \mu \mathrm{m}$ and then deactivated. The noise 389 indicates the time when the fiber was transferred between solutions. The sudden change in stress 390 indicates the stretch-release cycle performed to measure stiffness. The grey force trace indicates 391 the stress produced by the reference isometric contraction performed at the average SL of 2.6 $\mu \mathrm{m}$. 392 (A stiffness test was also performed before active shortening but not used in the analysis).

393 Figure 2

394 Electrophoresis of MHCs. Lanes 1-3: fibers on which the mechanical measurements were 395 performed. Lane 4: rabbit psoas muscle. Lane 5: rabbit muscles used as an electrophoretic 396 marker. Fibers in lanes 1 and 3 expressed MHC IId and were identified as Type II. The fiber in 397 lane 4 expressed MHC I and was identified as Type I.

$399 \quad$ Figure 3

400 Representative traces of the transient force changes during shortening of Type I and Type II 401 fibers. Force decreases during shortening in two phases characterized by a steep initial decrease 402 followed by a slower decrease in force fitted by two linear regressions (red and blue lines). The 403 slopes of the steep and slow phases are respectively S1 and S2. Type I fibers showed a 404 significantly steeper decay in force during the initial phase of shortening and lower S2 and force 
405 depression $(\mathrm{p}<0.05)$ than Type II fibers. Force depression was respectively 2 and $9 \%$ in Type I 406 and Type II fibers shown in this figure.

407

408 Figure 4

409 Force depression as a function of stiffness depression. There is a statistically significant 410 correlation between force depression and stiffness depression. Type II-0.115 and Type II-17\% 411 V0 refers to Type II fibers shortened at a constant speed of 0.115 fiber/s and $17 \% \mathrm{~V}_{0}$ 412 respectively.

413 Figure 5

414 Force depression as a function of S1. There is a statistically significant correlation between force 415 depression and stiffness depression. Type II-0.115 and Type II-17\% V0 refers to Type II fibers 416 shortened at a constant speed of 0.115 fiber/s and $17 \% \mathrm{~V}_{0}$ respectively. 


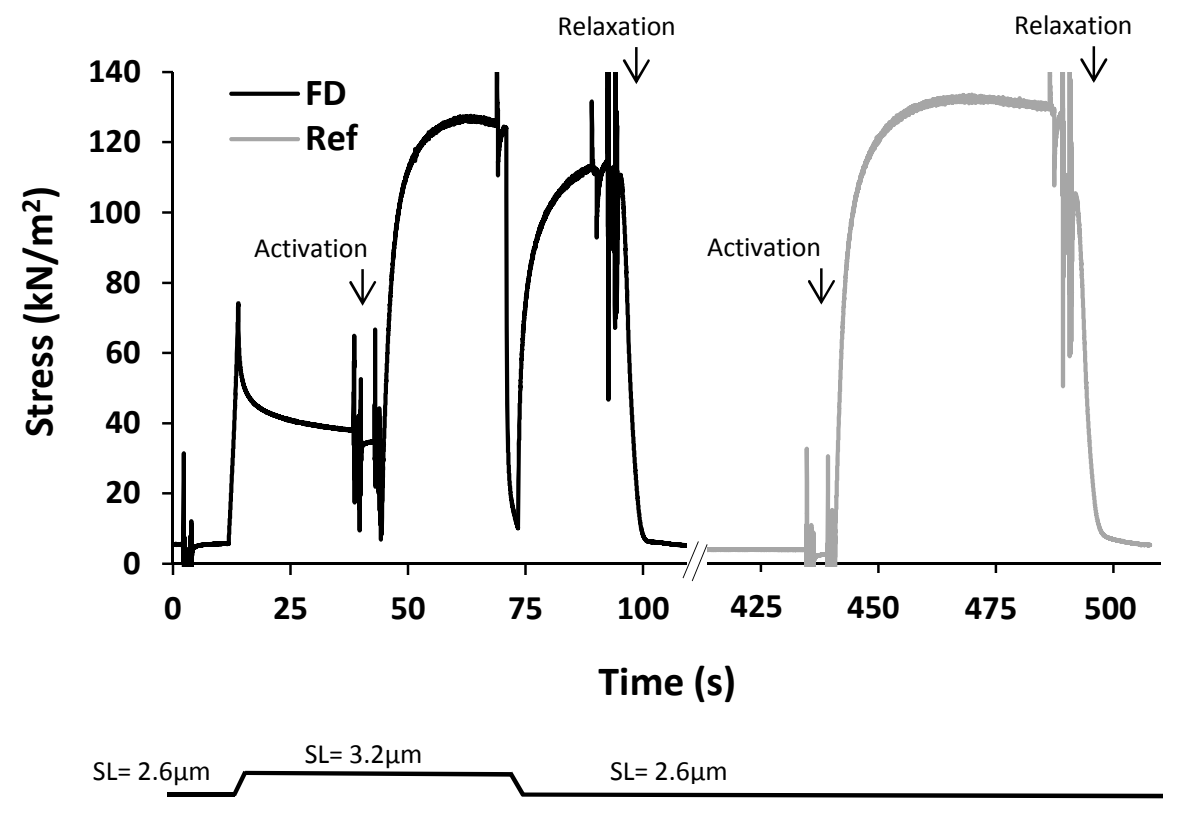


MHC Ila MHC Ild $\mathrm{MHC}$ Ilb $\mathrm{MHC}$ I 


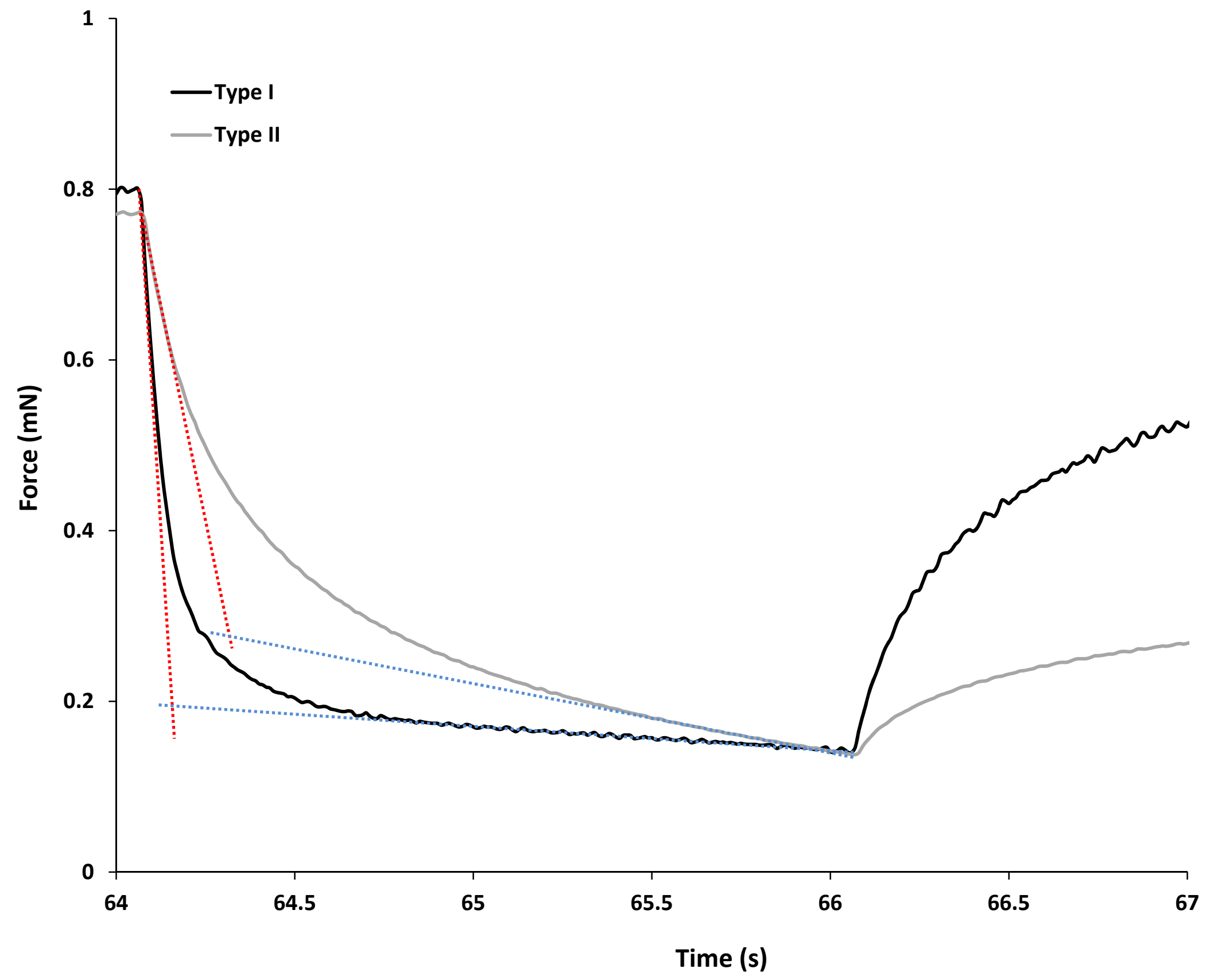




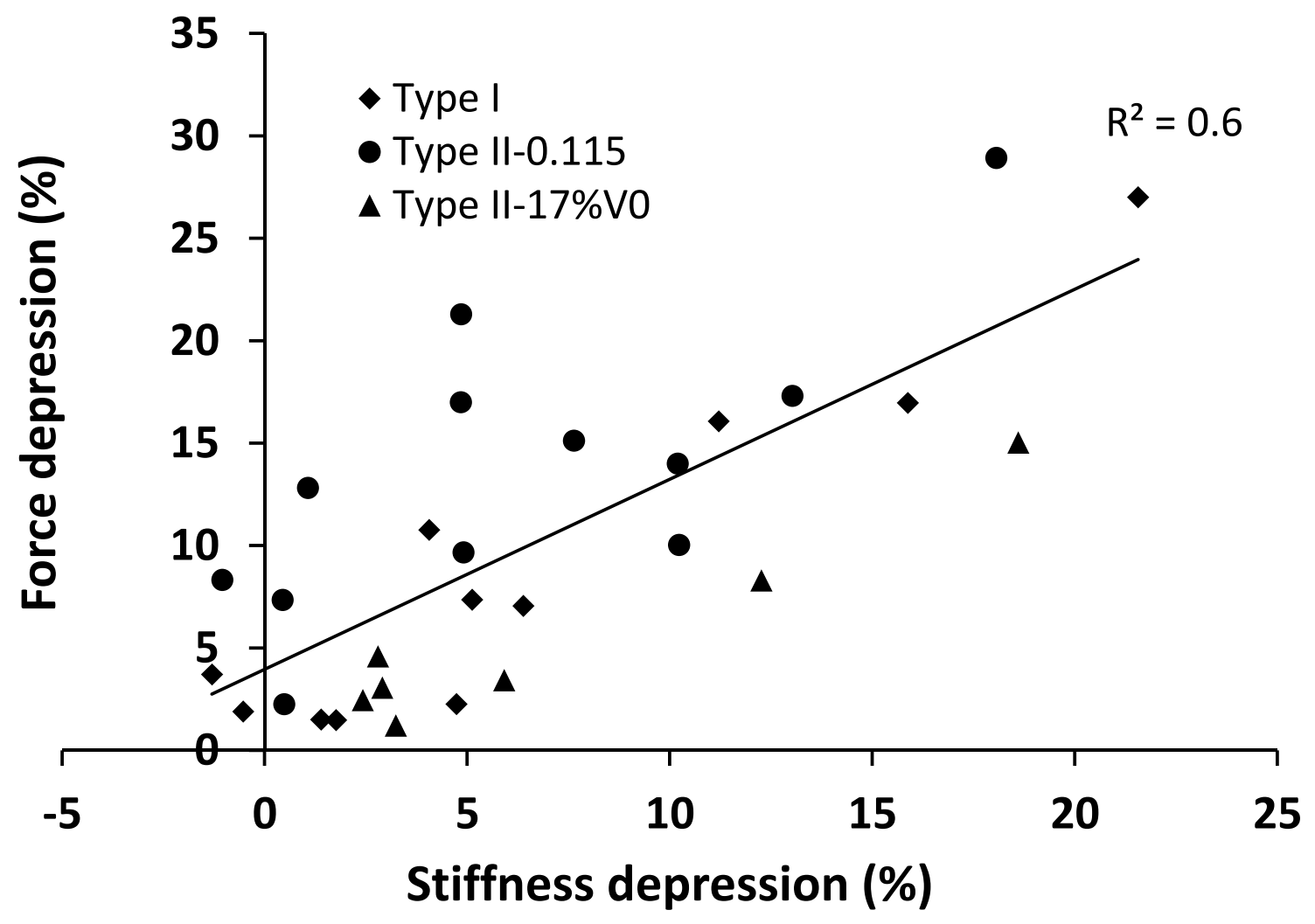




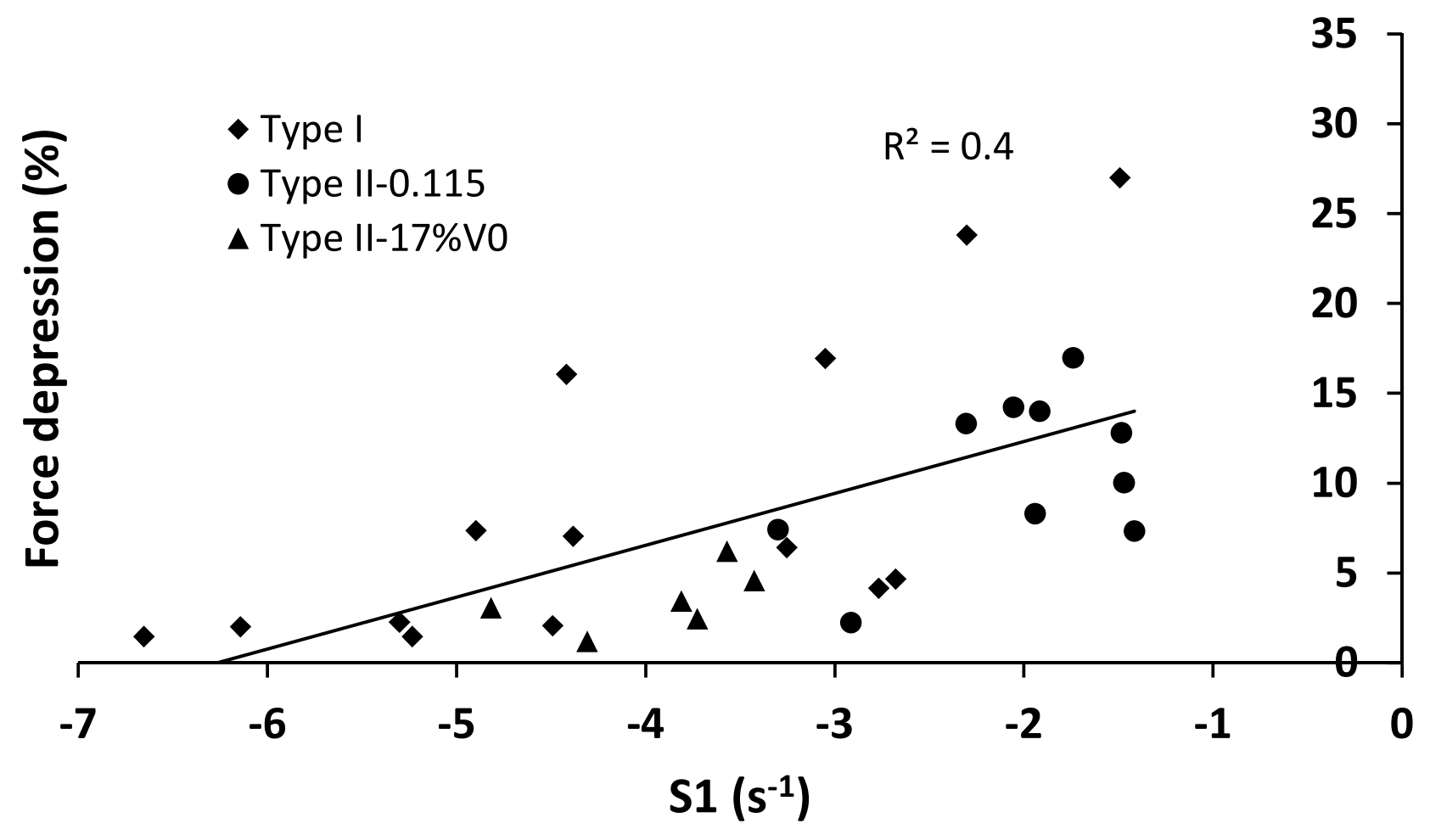

